\title{
Comunicação/Communication
}

\section{Controle da leishmaniose visceral no município de Porteirinha, Estado de Minas Gerais, no período de 1998 a 2003}

\author{
Control of visceral leishmaniasis in the town of Porteirinha, State of Minas Gerais, Brazil, from \\ 1998 to 2003
}

\section{Ricardo Andrade Barata ${ }^{1,2}$, João Carlos França da Silva ${ }^{3}$, Jaime Costa da Silva ${ }^{4}$, Saulo Neris de Almeida ${ }^{5}$, Luciana de Almeida Silva Teixeira ${ }^{6}$ e Edelberto Santos Dias ${ }^{2}$}

\begin{abstract}
RESUMO
Introdução: No município de Porteirinha, Estado de Minas Gerais, foram registrados 23 casos humanos de leishmaniose visceral (LV) nos anos de 1998 e 1999. Métodos: Foi realizado um estudo envolvendo a tríade de ações preconizadas no controle da LV. Pacientes com leishmaniose humana foram tratados e cães sorologicamente positivos foram eutanasiados, trimestralmente. $\mathrm{O}$ inseticida piretróide $\alpha$-cipermetrina foi aplicado nos bairros onde casos humanos foram registrados. Resultados: Houve uma redução da soroprevalência canina e de flebotomíneos capturados, após a implementação das medidas de controle, refletindo na diminuição de casos humanos de leishmaniose visceral. Conclusões: Os resultados mostraram a eficiência destas medidas quando empregadas em conjunto.
\end{abstract}

Palavras-chaves: Leishmaniose visceral. Medidas de controle. Porteirinha.

\begin{abstract}
Introduction. In the town of Porteirinha, State of Minas Gerais, 23 human cases of visceral leishmaniasis (VL) in 1998 and 1999 were recorded. Methods: A study was conducted involving the triad of action recommended for the control of VL. Patients were treated and serologically positive dogs were euthanized quarterly. The pyrethroid insecticide $a$-cypermethrin was applied in the neighborhoods where human cases were recorded. Results: A reduction in canine seroprevalence and sand flies occurred following the implementation of control measures, reflecting in a reduction in human cases of VL. Conclusions: The results show the efficiency of such control measures when used in association.
\end{abstract}

Keywords: Visceral leishmaniasis. Control measures. Porteirinha.

No Brasil, o controle da leishmaniose visceral (LV) tem sido baseado em um conjunto de ações (tratamento dos casos humanos, detecção/eliminação dos cães soropositivos e combate ao vetor com utilização de inseticidas residuais aspergidos no domicílio e anexos), associado ao manejo ambiental e à vigilância epidemiológica ${ }^{1}$. No entanto, trabalhos que demonstram a eficiência destas medidas

1. Laboratório de Parasitologia, Departamento de Ciências Biológicas, Universidade Federal dos Vales do Jequitinhonha e Mucuri, Diamantina, MG. 2. Laboratório de Leishmanioses, Centro de Pesquisas René Rachou, Fundação Oswaldo Cruz, Belo Horizonte, MG. 3. Laboratório de Parasitologia, Instituto de Ciências Biológicas, Universidade Federal de Minas Gerais, Belo Horizonte, MG. 4. Divisão de Vigilância Epidemiológica, Secretaria de Vigilância em Saúde, Brasília, DF. 5. Secretaria Municipal de Saúde de Porteirinha, Porteirinha, MG. 6. Departamento de Clínica Médica, Universidade Federal do Triângulo Mineiro, Uberaba, MG.

Endereço para correspondência: Dr. Ricardo Andrade Barata. Dept ${ }^{\circ}$ Ciências Biológicas/UFVJM. Campus JK, BR- 367, km 583, Alto da Jacuba, 39100-000

Diamantina, MG.

Tel: 5538 3532-1236

e-mail: ricbarata@hotmail.com

Recebido para publicação em 31/05/2010

Aceito em 26/11/2010 são escassos e pouco representativos, por não levarem em conta, as características peculiares dos elementos que compõem a cadeia epidemiológica, específicos para cada região.

No município de Porteirinha (latitude $15^{\circ} 44^{\prime} 42^{\prime}$ sul, longitude $43^{\circ} 01^{\prime} 46^{\prime}$ oeste), Estado de Minas Gerais, 23 casos humanos foram registrados nos anos de 1998 e 1999. Diante deste quadro, a partir de 2000, iniciamos um estudo visando conhecer o padrão ecoepidemiológico da LV na região, que foi caracterizado pela presença constante do vetor, alta prevalência canina e a ocorrência de casos humanos, traduzindo-se em um grave problema de saúde pública local ${ }^{2,3}$.

O presente trabalho avalia a efetividade do conjunto de ações, realizado no município de Porteirinha de acordo com o protocolo recomendado pelo Ministério da Saúde, no controle da LV, ou seja, o tratamento dos casos humanos, a detecção e eliminação de cães soropositivos e a aplicação de inseticida de poder residual contra o vetor.

Casos humanos de LV foram definidos a partir do quadro clínico e laboratorial compatíveis, associado à presença de parasita na medula óssea e/ou sorologia positiva. As reações sorológicas empregadas no diagnóstico foram o teste imunocromatográfico com antígeno rK39 (TRALd) e a reação de imunofluorescência indireta (RIFI) com títulos $\geq 1 / 40$. Os 23 pacientes positivos foram tratados com antimoniato de $\mathrm{N}$-metil-glucamina (Glucantime ${ }^{\circledR}$ ), anfotericina $\mathrm{B}$ ou associação entre as duas drogas, evoluindo para resolução completa do quadro. Os demais casos, identificados a partir de 2000, também, obedeceram aos procedimentos supracitados, sendo 6 casos no ano 2000, 2 casos em 2001, 3 casos em 2002 e 1 caso em 2003, que também tiveram resposta terapêutica satisfatória.

A detecção de cães soropositivos foi realizada durante os anos de 1998 e 1999. Agentes de saúde do município coletaram amostras de sangue de todos os cães domiciliados através de papel filtro e analisadas por imunofluorescência indireta (RIFI) ${ }^{4}$. O título discriminante de 1:40 foi usado de acordo com Costa cols ${ }^{5}$. A partir do quarto semestre de 2000, os cães soropositivos foram eutanasiados.

Entre novembro de 2002 e maio de 2003, armadilhas luminosas do tipo CDC foram expostas em três bairros eleitos para este estudo (São Judas, Vila União e Vila Vitória), levando-se em consideração a presença confirmada de casos humanos e a alta soroprevalência canina nestes bairros. Duas residências por bairro foram escolhidas para a exposição das armadilhas, sendo colocadas, no intra e peridomicílio, durante 5 dias consecutivos por mês, como realizado 
por Barata cols ${ }^{2}$. A partir de novembro de 2002, foram iniciadas as atividades de borrifação com o inseticida $\alpha$-cipermetrina no bairro Vila União e estendida para os bairros São Judas e Vila Vitória, nos meses subsequentes. $\mathrm{O}$ protocolo de borrifação foi realizado de acordo com o preconizado pelo Ministério da Saúde, atingindo as paredes internas e externas dos domicílios e anexos (galinheiros, estábulos, depósitos).

Para análise dos resultados, foi utilizado o teste $t$ de Student, não pareado e considerando como significativo $\mathrm{p}<0,05$. Os dados foram expressos como média \pm erro padrão da média (EPM).

No município de Porteirinha, observa-se que a maioria dos casos está presente em bairros situados nos pés de morro, formados por aglomerados subnormais, com a população vivendo em condições precárias, com baixo nível socioeconômico e presença de flebotomíneos e animais domésticos, como evidenciado por Barata cols $^{2}$ e França-Silva cols ${ }^{3}$.

Diversos esquemas terapêuticos têm sido adotados para o tratamento da leishmaniose visceral com resultados divergentes no que diz respeito à droga de escolha ${ }^{6,7}$. No entanto, neste estudo não houve qualquer divergência, sendo utilizado o antimoniato de $\mathrm{N}$-metil-glucamina (Glucantime ${ }^{\circ}$ ), associado ou não à anfotericina $\mathrm{B}$, com resposta terapêutica favorável ao final do tratamento em todos os pacientes atendidos entre os anos de 1998 e 2003.

A Figura 1 mostra o efeito da retirada sistemática de cães soropositivos nos bairros de alta prevalência no município de Porteirinha, entre o terceiro trimestre de 1998 e o terceiro trimestre de 2002. Observa-se um decréscimo estatisticamente significativo $(\mathrm{p}=0,0049)$ da prevalência canina, após a retirada sistemática de cães a partir do quarto trimestre de 2000 (Figura 2A), inclusive nos outros bairros do município (dados não publicados). Este resultado mostrou que a eutanásia de cães soropositivos pode diminuir a prevalência da LV canina, como também demonstrado por outros autores ${ }^{8,9}$.

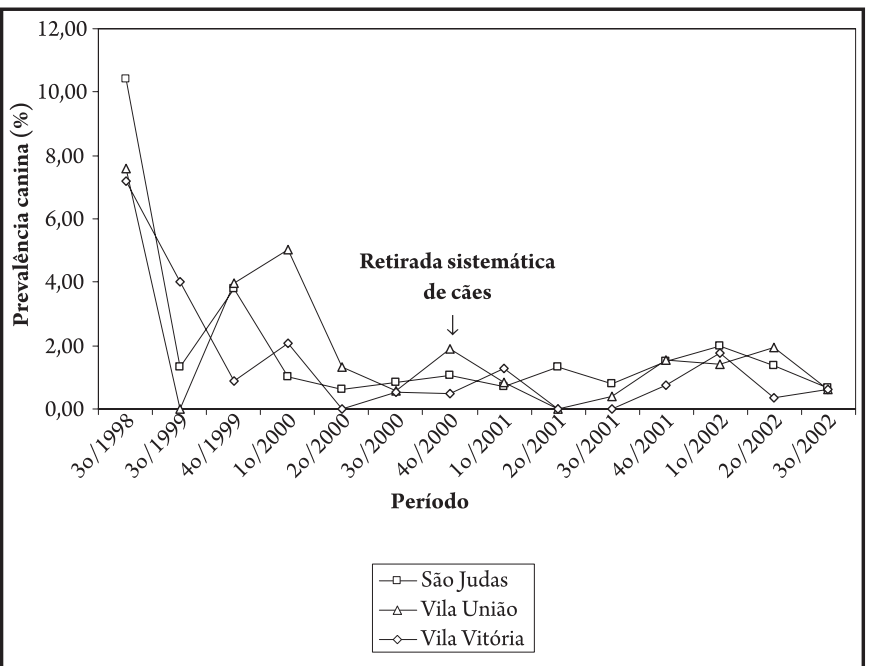

FIGURA 1 - Efeito da retirada sistemática de cães soropositivos para leishmaniose visceral nos bairros de alta prevalência no município de Porteirinha, entre 1998 a 2002.

Entre os anos de 1998 e 2002, houve uma redução considerável da prevalência de LV canina nos bairros analisados (redução de: 10,4 para 0,6 no bairro São Judas; 7,6 para 0,6 na Vila União e 7,2 para 0,6 na Vila Vitória) (Figura 1). Um estudo realizado no município de Montes Claros $(\mathrm{MG})^{10}$ mostrou que cães soropositivos foram eutanasiados, juntamente com a prática de borrifação. Os autores

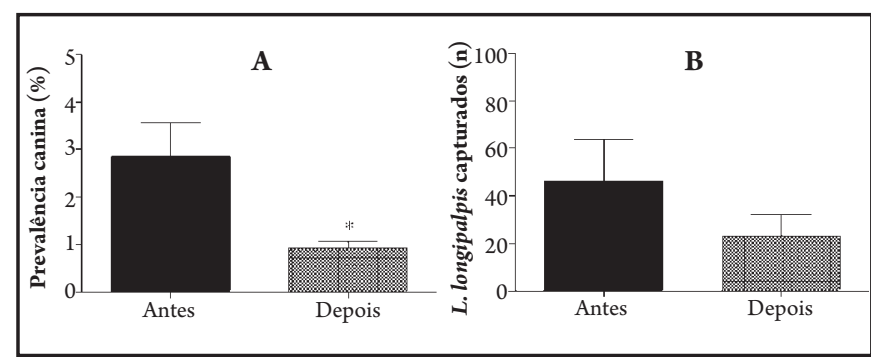

FIGURA 2 - Média da porcentagem de prevalência canina $(A)$ e do número de espécimes de Lutzomyia longipalpis capturados (B), antes e depois das medidas de intervenção realizadas no município de Porteirinha (MG), entre o quarto trimestre de 2000 e o primeiro trimestre de 2003.

${ }^{*} \mathrm{p}<0,05$ ( $t$ Student, não pareado).

observaram que a prevalência da LV canina reduziu em 50\% no município, sendo possível controlar a infecção canina, com auxílio do emprego do inseticida contra o vetor ${ }^{10}$. No presente estudo, após a retirada de cães, foi feita a aplicação com inseticida piretróide a-cipermetrina, nas residências e anexos, nos bairros onde foram notificados casos humanos.

A porcentagem de residências borrifadas nos bairros São Judas, Vila União e Vila Vitória foi em torno de 90\%. De um modo geral, o efeito da borrifação sobre a população de L. longipalpis não apresentou uma diferença estatisticamente significativa $(\mathrm{p}=0,233)$, apesar de observarmos uma tendência à redução do número de vetores capturados após o início da intervenção (Figura 2B). A Figura 3 mostra o efeito da borrifação sobre a densidade de Lutzomyia longipalpis por trimestre em cada bairro estudado. A realização de apenas um ciclo de borrifação nestes bairros e o fato de não termos atingido a totalidade de residências borrifadas pode explicar este resultado. Além disso, a falta de recursos humanos e financeiros, associada à resistência de alguns moradores durante o processo de aplicação do inseticida foram limitações enfrentados na realização desta ação de controle.

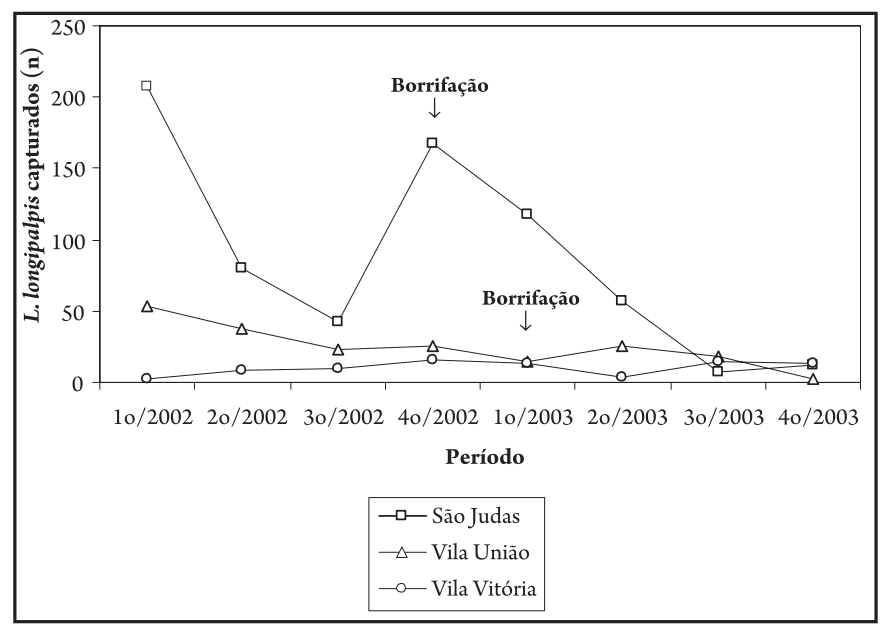

FIGURA 3 - Efeito da borrifação sobre o número de espécimes de Lutzomyia longipalpis capturados por trimestre, nos bairros com casos de leishmaniose visceral humana, no município de Porteirinha (2002-2003).

Muitos autores têm registrado que a aplicação de inseticida residual reduz a densidade de flebotomíneos e, consequentemente, a incidência da doença em várias localidades ${ }^{11-13}$. De fato, se considerarmos o número de casos humanos ao longo deste estudo percebemos um declínio considerável. No entanto, atribuímos ao Programa de Controle da Leishmaniose Visceral, o sucesso deste 
estudo realizado no município de Porteirinha, baseado não somente na aplicação do inseticida residual, mas também no tratamento dos casos humanos e eliminação dos cães soropositivos.

Diante disso, manifestamos apenas a preocupação sobre a necessidade de uma rigorosa vigilância epidemiológica a fim de se evitar a reinstalação de novos focos no município. A educação sanitária e a participação da comunidade constituem fatores fundamentais nos programas de controle, requisitos primordiais para o desenho de estratégias ou para a definição de metodologias específicas para cada área endêmica.

\section{AGRADECIMENTOS}

A Jeová Vitor dos Santos, Simone Resende Marrocos e à Cynthia Fernandes Ferreira Santos.

\section{CONFLITO DE INTERESSE}

Os autores declaram não haver nenhum tipo de conflito de interesse no desenvolvimento do estudo.

\section{SUPORTE FINANCEIRO}

Fundação de Amparo à Pesquisa do Estado de Minas Gerais (FAPEMIG).

\section{REFERÊNCIAS}

1. Ministério da Saúde. Manual de vigilância e controle da leishmaniose visceral. Brasília: Secretaria de Vigilância em Saúde. Departamento de Vigilância Epidemiológica; 2006.

2. Barata RA, França-Silva JC, Fortes-Dias CL, Costa RT, Silva JC, Vieira EP, et al. Phlebotomines sand flies in Porteirinha, an endemic area of American visceral leishmaniasis in the State of Minas Gerais, Brazil. Mem Inst Oswaldo Cruz 2004; 99:481-487.

3. França-Silva JC, Barata RA, Costa RT, Michalsky EM, Machado-Coelho GLL, Vieira EP, et al. Importance of Lutzomyia longipalpis in the dynamics of transmission of canine visceral leishmaniasis in the endemic area of Porteirinha Municipality, Minas Gerais, Brazil. Vet Parasitology 2005; 131: 213-220.

4. Camargo ME, Rebonato C. Cross-reactivity in fluorescence tests for Trypanosoma and Leishmania antibodies. Am J Trop Med Hyg 1969; 18:500-505.

5. Costa RT, Magalhães-Rocha MN, Mayrink W. Leishmaniose visceral canina: avaliação da metodologia sorológica utilizada em inquéritos epidemiológicos. Rev Soc Bras Med Trop 1991; 24:21-25.

6. Kafetzis DA, Velissariou IM, Stabouli S, Mavrikou M, Delis D, Liapi G. Treatment of paediatric visceral leishmaniasis: amphotericin B or pentavalent antimony compounds? Int J Antimicrob Agents 2005; 25:26-30.

7. Minodier P, Robert S, Retornaz K, Garnier JM. Visceral leishmaniasis: new drugs. Arch Pediatr 2003; 10:550-556.

8. Genaro O. Leishmaniose visceral canina experimental. [Tese]. [Belo Horizonte]: Universidade Federal de Minas Gerais; 1993.

9. Magalhães PA, Mayrink W, Costa CA, Melo MN, Dias M, Batista SM, et al. Calazar na zona do Rio Doce-Minas Gerais. Resultados de medidas profiláticas. Rev Inst Med Trop 1980; 22:197-202.

10. França-Silva JC, Costa RT, Siqueira AM, Machado-Coelho GLL, Costa CA, Mayrink W, et al. Epidemiology of canine visceral leishmaniosis in the endemic area of Montes Claros Municipality, Minas Gerais State, Brazil. Vet Parasitol 2003; 111:161-173.

11. Le Pont F, Mariscal-Padilla J, Desjeux P, Richard A, Mouchet J. Impact de pulverisation de deltamethrine dans la leishmaniose de Bolivie. Ann Soc Belge Med Trop 1989; 69:223-232.
12. Falcão AL, Falcão AR, Pinto CT, Gontijo CMF, Falqueto A. Effect of deltamethrin spraying on the sandfly populations in a focus of American Cutaneous Leishmaniasis. Mem Inst Oswaldo Cruz 1991; 86:399-404.

13. Oliveira-Filho AM, Melo MTV. Vectors control importance on leishmaniasis transmission. Mem Inst Oswaldo Cruz 1994; 89:451-456. 